\title{
Recurrent giant cranial desmoid tumor in a 3-year-old boy with familial adenomatous polyposis requiring bifrontoparietal cranioplasty: case report
}

\author{
Luyuan Li, BA, John N. Jensen, MD, ${ }^{2}$ Sara Szabo, MD, PhD, ${ }^{3}$ Peter VanTuinen, $\mathrm{PhD},{ }^{3}$ and \\ Sean M. Lew, MD1
}

Departments of ${ }^{1}$ Neurological Surgery and ${ }^{2}$ Plastic Surgery, Medical College of Wisconsin/Children's Hospital of Wisconsin, Milwaukee, Wisconsin; and '3Department of Pathology, Cincinnati Children's Hospital Medical Center, Cincinnati, Ohio

\begin{abstract}
Desmoid tumors, also known as aggressive fibromatosis, are locally infiltrating musculoaponeurotic neoplasms arising in connective tissues. Desmoid tumors may be associated with familial adenomatous polyposis (FAP), a genetic disorder that presents with hundreds to thousands of precancerous colorectal polyps. The authors report the case of an 18-month-old boy who underwent resection of a right temporal desmoid tumor (initially diagnosed as cranial fasciitis) and developed a bilateral frontoparietal calvarial desmoid tumor 2 years later. The patient underwent gross-total resection of the tumor that required a large cranioplasty. He was subsequently diagnosed with FAP. The patient has been without tumor recurrence for 9 years afterwards and has not required revision of his cranioplasty. This is the first report describing a recurrent cranial desmoid tumor in a pediatric patient with FAP. The authors believe, however, that some of the cases previously reported as cranial fasciitis are likely desmoid tumors pathobiologically and genetically.
\end{abstract}

http://thejns.org/doi/abs/10.3171/2016.6.PEDS15741

KEY WORDS adenomatous polyposis coli; aggressive fibromatosis; cranioplasty; desmoid tumor; familial adenomatous polyposis; oncology

$\mathrm{F}$ AMILIAL adenomatous polyposis (FAP) is an autosomal dominant disorder that is caused by a loss of function mutation of the adenomatous polyposis coli $(A P C)$ gene located at the $\mathrm{q}$ arm of chromosome 5. APC protein acts as a tumor suppressor, degrading oncogenic transcription factor $\beta$-catenin. Thus $A P C$ mutation can lead to tumor formation and high levels of $\beta$-catenin in the cell nucleus. ${ }^{12}$

Patients with FAP can develop hundreds of colorectal polyps and desmoid tumors, which are locally infiltrating musculoaponeurotic neoplasms arising in connective tissues. ${ }^{8}$ Desmoid tumors occur in $10 \%-15 \%$ of patients with FAP. ${ }^{11}$ Histologically, desmoid tumors consist of fibroblastlike lesional cells, in an infiltrative growth pattern, embedded within a collagen network. Nuclear staining for $\beta$ catenin is noted in $67 \%-80 \%$ of cases. ${ }^{7}$ Desmoid tumors can be either abdominal or, less commonly, extraabdominal. ${ }^{4}$ Although desmoid tumors do not metastasize, they can become locally aggressive, infiltrating and destroying adjacent organs. Desmoid tumor is the second leading cause of death in patients with FAP, following colorectal carcinoma. ${ }^{1}$

Two cases of primary cranial desmoid tumors associated with FAP had been previously reported. ${ }^{9,26}$ We report the case of an 18-month-old boy who underwent grosstotal resection of a right temporal desmoid tumor, which was initially diagnosed as cranial fasciitis. Two years later, he developed another bifrontoparietal desmoid tumor and was subsequently diagnosed with FAP. This is the first report describing recurrent primary cranial desmoid tumor in a pediatric patient with FAP. We believe, however, that some of the cases previously reported as cranial fasciitis are likely desmoid tumors pathobiologically and genetically, as also contemplated by others, ${ }^{19}$ and such a diagnosis should be considered in future clinical practices for lesional management and FAP risk assessment. The recur-

ABBREVIATIONS APC = adenomatous polyposis coli; $F A P=$ familial adenomatous polyposis; $P M M A=$ polymethylmethacrylate .

SUBMITTED December 27, 2015. ACCEPTED June 17, 2016.

INCLUDE WHEN CITING Published online September 16, 2016; DOI: 10.3171/2016.6.PEDS15741. 


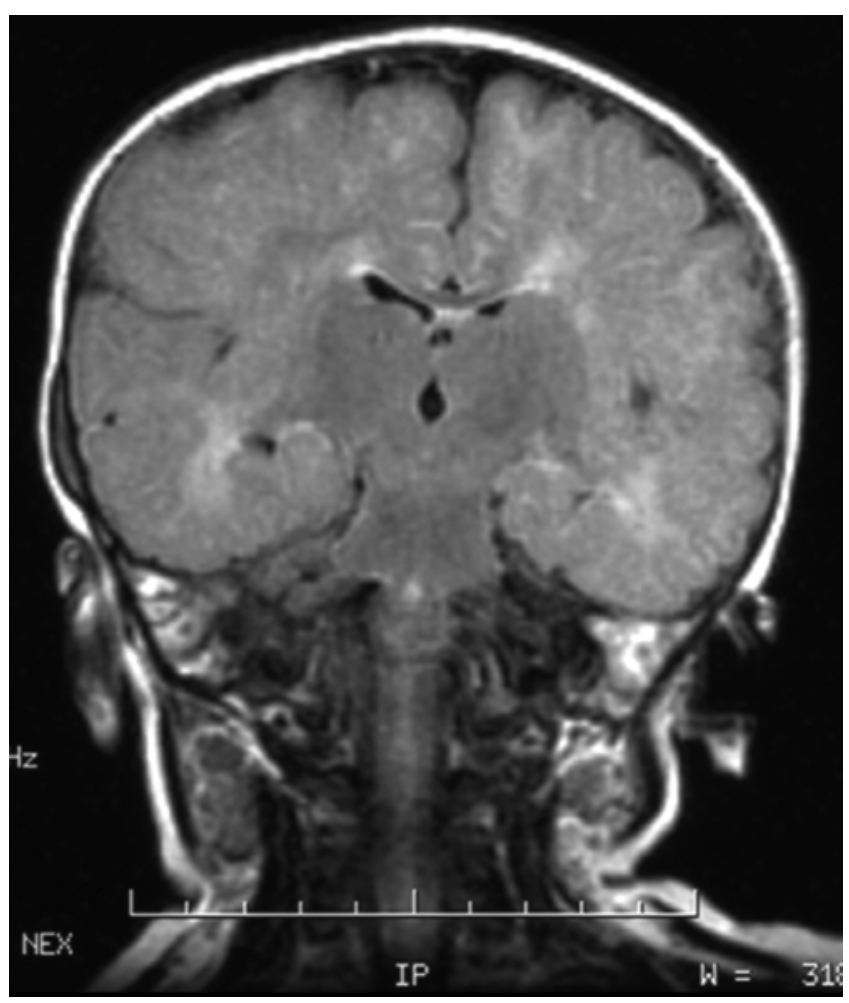

FIG. 1. Coronal T1-weighted MR image of the brain at 15 months of age demonstrating the original right temporal mass.

rence of desmoid tumor emphasizes the need for careful follow-up after desmoid tumor excision in pediatric patients with FAP.

\section{Case Report}

History and Initial Examination

An 18-month-old boy initially presented with a right temporal mass, which had been slowly growing over the prior several months (Fig. 1). His medical history was significant for premature birth at 32 weeks, patent ductus arteriosus, and microcephaly. At presentation, the physical examination showed a firm $7 \times 5 \mathrm{~cm}$ temporal mass and hypotonia. A postcontrast CT scan demonstrated a right extracranial soft-tissue mass involving the squamosal portions of the temporal bone. The patient subsequently underwent resection of the lesion. Intraoperatively, the abnormal mass was excised circumferentially along with the attached periosteum; the underlying skull had multiple eroded areas with a honeycomb appearance. Total resection of the tumor was achieved and the eroded bone underneath was curetted to normal appearing bone. Histopathological analysis showed a proliferation of spindled mesenchymal cells with a fascicular pattern. The lesion was initially diagnosed as cranial fasciitis, but was later determined to be desmoid tumor as immunohistochemical analysis showed nuclear localization of $\beta$-catenin (Fig. 2).

\section{Second Presentation and Examination}

The patient was subsequently lost to follow-up. Two years after the initial operation (age 3 years), the patient

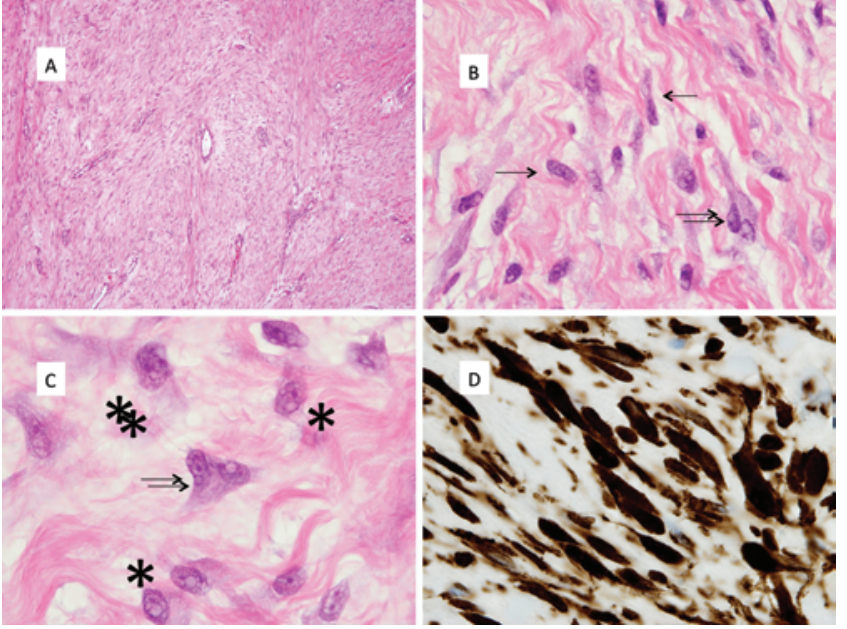

FIG. 2. Photomicrographs. A: A fibromatous fascicular to storiform low-power appearance is imparted by a predominance of extracellular matrix with interspersed delicate small blood vessels. $\mathrm{H}$ \& $\mathrm{E}$, original magnification $\times 20$. B: The predominantly bland spindled mesenchymal cells with sharply demarcated nuclei and small amount of relatively indistinct cytoplasm (arrows) are scattered in abundant collagen-rich matrix, along with delicate to compact hypereosinophilic wavy collagen fibers. $H \& E$, original magnification $\times 100$. C: Regionally slightly atypical cells are interspersed, with bilobed nuclei (arrows), plumper vesiculated nuclei, and tiny but prominent nucleoli (single asterisks) in delicate stellate cytoplasm with often indistinct cytoplasmic borders (double asterisks). $H \& E$, original magnification $\times 400$. D: The strong nuclear positivity and lighter cytoplasmic positivity for $\beta$-catenin (immunohistochemistry) are salient supportive features for this diagnosis of desmoid tumor. Original magnification $\times 100$. Figure is available in color online only.

presented with a new cranial mass. A physical examination demonstrated a large, nontender, bilateral frontoparietal mass, hypotonia, and left esotropia with intermittent disconjugate gaze. These deficits were believed to be due to long-standing intracranial hypertension. Initially the patient was thought to not be MRI compatible due to a cardiac implant used for treatment of his patent ductus arteriosus (after his second tumor resection his implant was deemed MRI compatible). A postcontrast head CT scan showed a heterogeneously enhancing solid mass involving the frontoparietal regions with extensive bone destruction (Fig. 3). The mass also extended into the epidural space and severely compressed the underlying cerebrum, but the superior sagittal sinus appeared patent. Further staging workup with a radionuclide bone scan and CT scan of the chest, abdomen, and pelvis showed no evidence of extracranial abnormalities.

\section{Operation}

The patient underwent a limited biopsy to establish a tissue diagnosis. Histopathological examination revealed dense proliferation of small and elongated fibroblast-like cells with features suggestive of a desmoid tumor; immunohistochemical analysis demonstrated nuclear accumulation of $\beta$-catenin (Fig. 4). Fluorescence in-situ hybridization on explanted tumor cell culture and normal tissue showed deletion of bacterial artificial chromosome clone RP11-13O21 near the APC gene locus on chromosome $5 q 22.2$. This result supported a diagnosis of FAP. Nine 

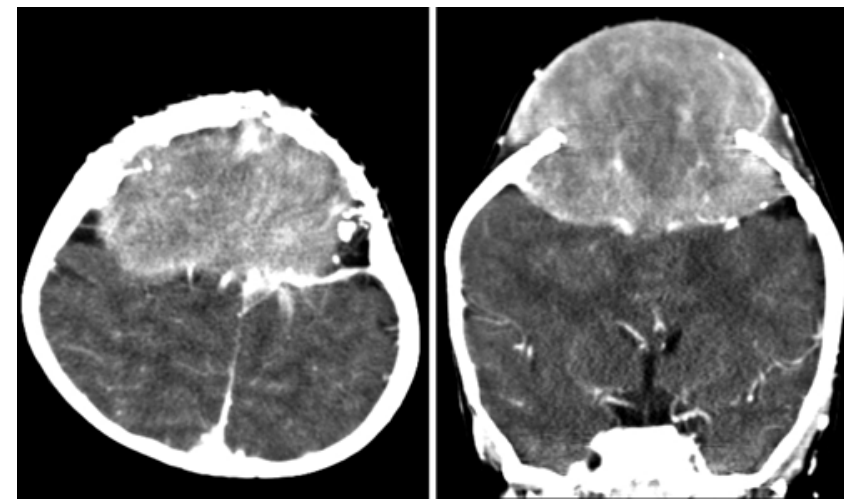

FIG. 3. Axial (left) and coronal (right) postcontrast cranial CT scans showing an extracranial and extradural heterogeneous solid mass (measuring $10 \times 6 \times 6 \mathrm{~cm}$ ) with aggressive bone destruction.

days after the biopsy, the patient underwent a complete resection with subsequent cranioplasty. Intraoperatively, the tumor was found to be densely adherent to the underlying dura in most areas. It overlaid, but did not invade, the superior sagittal sinus. Aggressive bipolar cautery was used to help create the plane. After the bulk of the tumor was resected, remnant tumor tissue was scraped and peeled off the dura and cauterized where it was believed to not be safe to remove (Fig. 5). This was also performed over the sinus with great care. Following the resection, the calvarial defect was repaired using a custom-made polymethylmethacrylate (PMMA) implant (Stryker).

\section{Postoperative Course}

Due to the long-standing extradural compression, the boy had a large epidural space following surgery. Two weeks after surgery he underwent placement of a subgaleal drain for a persistent subgaleal fluid collection that was believed to be a resolving hematoma or seroma. Following

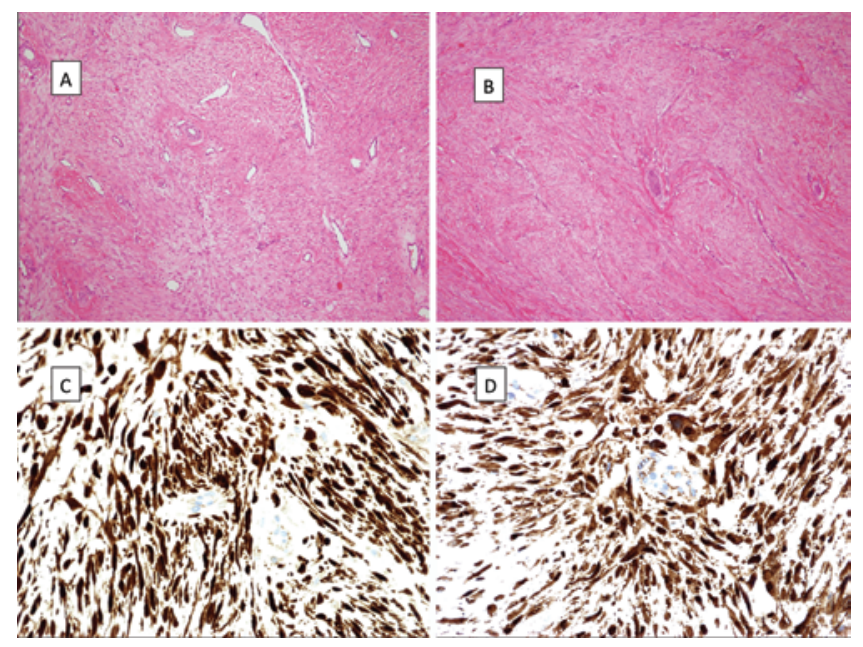

FIG. 4. Photomicrographs showing histopathological comparisons. Spindle-shaped fibroblasts in a collagen-rich matrix seen in first (A) and second (B) lesions. Nuclear positivity for $\beta$-catenin seen in first (C) and second $(D)$ lesions. $H \& E$ ( $A$ and $B$ ), immunostain for $\beta$-catenin ( $C$ and D). Original magnification $\times 20(A$ and $B), \times 100(C$ and $D)$. Figure is available in color online only.
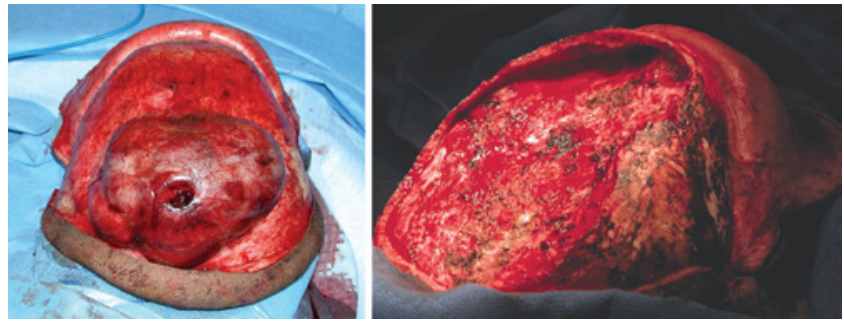

FIG. 5. Intraoperative images showing a superior view of the tumor exposed via a bicoronal incision (left); excision of the tumor and involved bone left a large cranial defect and flattened dura over the cerebrum (right). Figure is available in color online only.

drain placement he showed left upper-extremity weakness and imaging revealed a small dural defect with corresponding brain herniation. This was repaired, and he ultimately required a temporary lumboperitoneal shunt to address persistent CSF accumulation in the subgaleal space.

Nine years following the resection of the desmoid tumor, the patient continues to be developmentally delayed with left esotropia and disconjugate gaze. He has contour irregularities in the forehead region but the cranial implant continues to be satisfactory. Follow-up imaging has not shown any evidence of recurrence (Fig. 6). He ultimately required a total colectomy at the age of 12 years for extensive polyp disease.

\section{Discussion}

Desmoid tumors are histopathologically deceptively bland, but locally aggressive, soft-tissue neoplasms. Nuclear staining for $\beta$-catenin is noted in $67 \%-80 \%$ of cases. Desmoid tumors have a propensity for local recurrence after incomplete excision and for accelerated growth after trauma (such as incisional biopsy). Ten percent to $15 \%$ of patients with FAP have been found to have desmoid tumors, an incidence 850 times greater than that observed in the general population. ${ }^{11}$ When arising on the skull, desmoid tumor may erode into the underlying calvaria and disrupt associated CNS development in pediatric patients due to the mass effect of the tumor.

Desmoid tumor usually presents with a rapidly growing nontender mass, ${ }^{10}$ mimicking cranial fasciitis, a benign fi-
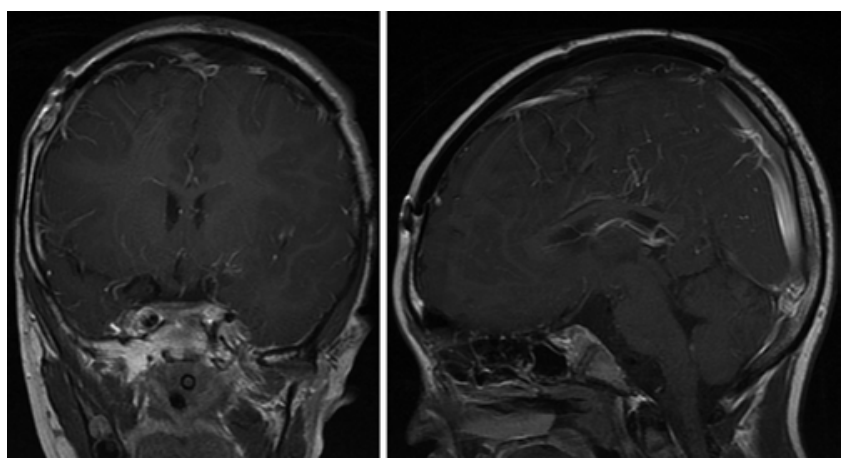

FIG. 6. Coronal (left) and sagittal (right) postcontrast T1-weighted MR images of the brain (4.5 years postoperatively) demonstrate a small epidural collection underneath the cranioplasty without evidence of residual or recurrent tumor. 
broblast lesion that rarely recurs. In fact, during the era prior to the use of $\beta$-catenin staining, rapidly growing infantile lesions of the scalp were most likely diagnosed as cranial fasciitis based on spindle cell morphology alone. Since the introduction of $\beta$-catenin staining there has been a shift to categorize similar cases - which showed nuclear positivity for $\beta$-catenin-as desmoid tumors. In this case, the first right temporal lesion was initially misdiagnosed as cranial fasciitis, and later confirmed to be a primary desmoid tumor based on the history of FAP and nuclear localization of $\beta$-catenin noted in immunohistochemical analysis. The second bifrontoparietal lesion is also more likely to be an independent primary desmoid tumor, as it did not overlap with the first lesion.

The management of desmoid tumors can be complex, involving surgery, systemic chemotherapy, and/ or radiation..$^{14}$ No clinical trials have been performed to compare the operative and nonoperative treatment of desmoid tumor. Radiation and chemotherapy have some success in improving the local control of desmoid tumor in adults. ${ }^{25}$ A regimen consisting of vinblastine and methotrexate showed a $31 \%$ response rate (complete or partial regression) for desmoid tumors in children..$^{22}$ High-dose tamoxifen and sulindac have also been found to be effective in treating desmoid tumor. ${ }^{15}$ Radiotherapy has been incorporated into the management of extraabdominal desmoid tumors, either as primary or adjuvant treatments. However, one study demonstrated that younger children, who were treated with radiotherapy, had higher recurrence rates. ${ }^{21}$ Furthermore, the CNS of younger children is more susceptible to the side effects of radiation and cytotoxic chemotherapy. ${ }^{24}$ Surgery is the first-line therapy for an extraabdominal desmoid tumor when it can be accomplished without significant functional impairment. ${ }^{18}$ Resection with histologically free margins remains an effective treatment for desmoid tumor in terms of long-term event-free survival, with a $22 \%-27 \%$ chance of recurrence after margin-negative resection. ${ }^{3,23}$ If margins are positive following resection, radiation therapy or chemotherapy may help maintain local control of tumor. ${ }^{13}$

Bone involvement by tumor is a very common cause of cranial defects. Repair of cranial defects improves cosmesis and decreases the incidence of epilepsy. ${ }^{20}$ There are many types of cranioplasty materials, such as autologous bone graft, titanium, and PMMA. When performing cranioplasty in young children, one important factor to consider is the continued skull growth with age. Autologous bone graft is widely used in the pediatric population due to its ability to grow into surrounding skull over time. ${ }^{17}$ Finding an appropriate donor site can be challenging, particularly in younger children in whom splitting the calvaria can be difficult. Titanium is commonly used in adults with a lower risk of infection, ${ }^{16}$ but it is hard to shape and does not integrate with growing skull well. PMMA remains among the most extensively used cranioplasty materials. It can be custom-made preoperatively or shaped intraoperatively. Compared with metals, PMMA is easier to shape and lighter in weight. ${ }^{2}$ Bone can grow into PMMA, resulting in a better fixation. ${ }^{6}$ The main complication of PMMA is infection, especially in patients undergoing postoperative radiation therapy. ${ }^{5}$ In the presented
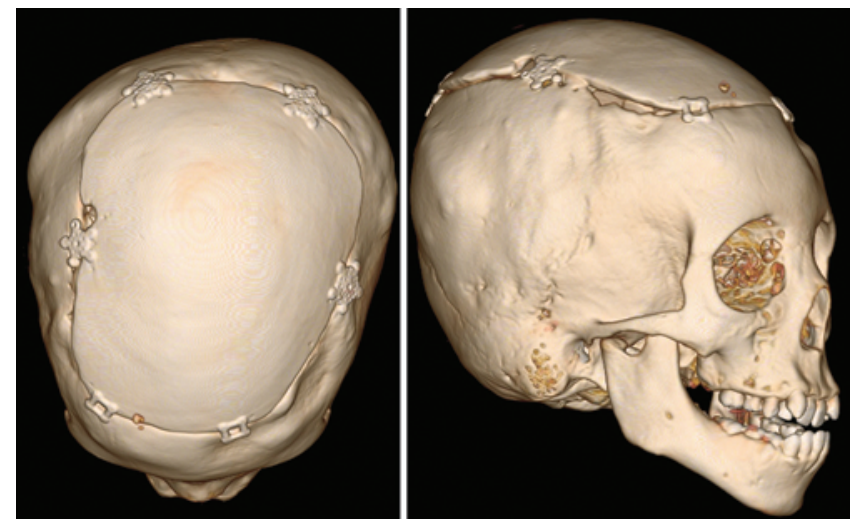

FIG. 7. Superior (left) and right anterolateral (right) views of 3D head CT at age 11 years (8 years postoperatively). Figure is available in color online only.

case, the large PMMA implant was placed at the age of 3 years and has not required replacement in the subsequent 9 years of follow-up. The segment of bone removed was limited in its depth of contour, and because the shape of this area changes little with growth, the implant placed in early childhood remained adequate even with extensive growth; that is, the growth of the intracranial space was adequately maintained by the untouched lateral, anterior, and posterior bone segments of the skull. At this point it is unlikely that he will require an implant revision (Figs. 7 and 8).

This case emphasizes the importance of frequent follow-up after excision of desmoid tumors in pediatric patients with FAP. Reports of recurrent cranial desmoid tumor formation appear to be rare in the literature. The patient would have benefited from closer follow-up and earlier removal of the tumor.

Desmoid tumor should be considered in the differential diagnosis of scalp and calvarial masses in infants and young children. Although not metastatic, desmoid tumors can cause local destruction due to their infiltrative property. Complete excision is required to minimize the risk

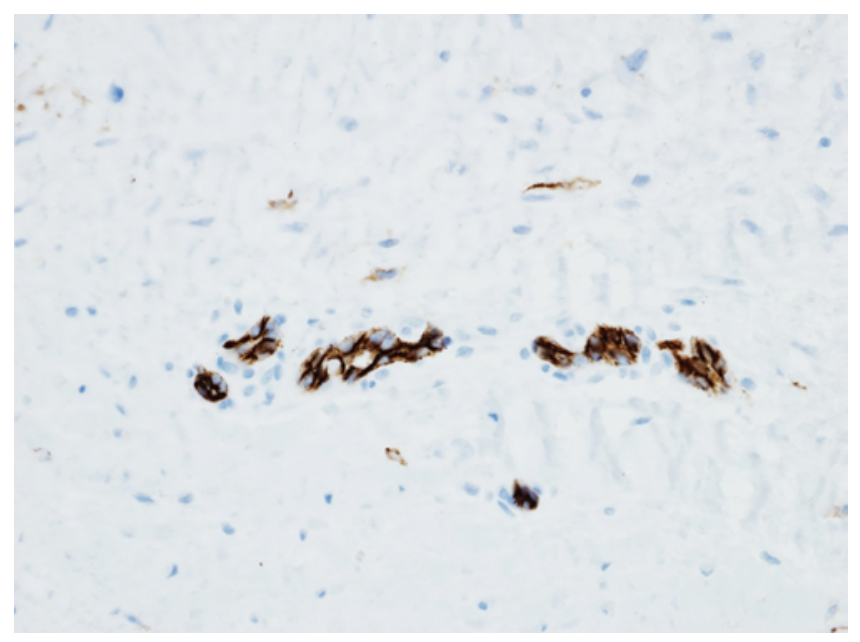

FIG. 8. Cells of the second lesion are negative for CD34 immunohistochemical stains with appropriate endothelial staining of vessels. Original magnification $\times 400$. Figure is available in color online only. 
of recurrence. In the pediatric population, a desmoid tumor may be the first lesion indicating a diagnosis of FAP. Patients with such predisposition should be followed up closely after surgical excision of desmoid tumors. A large vertex cranial defect in a toddler poses a difficult problem. We demonstrated that a PMMA implant eliminates the need for future revision when used to repair large cranial defects in a toddler.

\section{References}

1. Arvanitis ML, Jagelman DG, Fazio VW, Lavery IC, McGannon E: Mortality in patients with familial adenomatous polyposis. Dis Colon Rectum 33:639-642, 1990

2. Aydin S, Kucukyuruk B, Abuzayed B, Aydin S, Sanus GZ: Cranioplasty: Review of materials and techniques. J Neurosci Rural Pract 2:162-167, 2011

3. Ballo MT, Zagars GK, Pollack A, Pisters PW, Pollack RA: Desmoid tumor: prognostic factors and outcome after surgery, radiation therapy, or combined surgery and radiation therapy. J Clin Oncol 17:158-167, 1999

4. Bertario L, Russo A, Sala P, Eboli M, Giarola M, D'amico F, et al: Genotype and phenotype factors as determinants of desmoid tumors in patients with familial adenomatous polyposis. Int J Cancer 95:102-107, 2001

5. Blum KS, Schneider SJ, Rosenthal AD: Methyl methacrylate cranioplasty in children: long-term results. Pediatr Neurosurg 26:33-35, 1997

6. Bruens ML, Pieterman H, de Wijn JR, Vaandrager JM: Porous polymethylmethacrylate as bone substitute in the craniofacial area. J Craniofac Surg 14:63-68, 2003

7. Carlson JW, Fletcher CD: Immunohistochemistry for betacatenin in the differential diagnosis of spindle cell lesions: analysis of a series and review of the literature. Histopathology 51:509-514, 2007

8. de Oliveira Ribas M, Martins WD, de Sousa MH, de Aguiar Koubik AC, Avila LF, Zanferrari FL, et al: Oral and maxillofacial manifestations of familial adenomatous polyposis (Gardner's syndrome): a report of two cases. J Contemp Dent Pract 10:82-90, 2009

9. de Silva DC, Wright MF, Stevenson DA, Clark C, Gray ES, Holmes JD, et al: Cranial desmoid tumor associated with homozygous inactivation of the adenomatous polyposis coli gene in a 2-year-old girl with familial adenomatous polyposis. Cancer 77:972-976, 1996

10. Garza L, Allen L, Eghbalieh N, Palacios E, Neitzschman H: Cranial fasciitis of childhood: a lytic skull lesion. J La State Med Soc 164:347-349, 2012

11. Gurbuz AK, Giardiello FM, Petersen GM, Krush AJ, Offerhaus GJ, Booker SV, et al: Desmoid tumours in familial adenomatous polyposis. Gut 35:377-381, 1994

12. Henderson BR: Nuclear-cytoplasmic shuttling of APC regulates beta-catenin subcellular localization and turnover. Nat Cell Biol 2:653-660, 2000

13. Honeyman JN, Quaglia MP: Desmoid tumors in the pediatric population. Cancers (Basel) 4:295-306, 2012

14. Honeyman JN, Theilen TM, Knowles MA, McGlynn MM, Hameed M, Meyers P, et al: Desmoid fibromatosis in children and adolescents: a conservative approach to management. J Pediatr Surg 48:62-66, 2013
15. Janinis J, Patriki M, Vini L, Aravantinos G, Whelan JS: The pharmacological treatment of aggressive fibromatosis: a systematic review. Ann Oncol 14:181-190, 2003

16. Kuttenberger JJ, Hardt N: Long-term results following reconstruction of craniofacial defects with titanium micro-mesh systems. J Craniomaxillofac Surg 29:75-81, 2001

17. Moreira-Gonzalez A, Jackson IT, Miyawaki T, Barakat K, DiNick V: Clinical outcome in cranioplasty: critical review in long-term follow-up. J Craniofac Surg 14:144-153, 2003

18. Nieuwenhuis MH, Mathus-Vliegen EM, Baeten CG, Nagengast FM, van der Bijl J, van Dalsen AD, et al: Evaluation of management of desmoid tumours associated with familial adenomatous polyposis in Dutch patients. Br J Cancer 104:37-42, 2011

19. Rakheja D, Cunningham JC, Mitui M, Patel AS, Tomlinson GE, Weinberg AG: A subset of cranial fasciitis is associated with dysregulation of the Wnt/beta-catenin pathway. Mod Pathol 21:1330-1336, 2008

20. Rish BL, Dillon JD, Meirowsky AM, Caveness WF, Mohr JP, Kistler JP, et al: Cranioplasty: a review of 1030 cases of penetrating head injury. Neurosurgery 4:381-385, 1979

21. Rutenberg MS, Indelicato DJ, Knapik JA, Lagmay JP, Morris C, Zlotecki RA, et al: External-beam radiotherapy for pediatric and young adult desmoid tumors. Pediatr Blood Cancer 57:435-442, 2011

22. Skapek SX, Ferguson WS, Granowetter L, Devidas M, PerezAtayde AR, Dehner LP, et al: Vinblastine and methotrexate for desmoid fibromatosis in children: results of a Pediatric Oncology Group Phase II Trial. J Clin Oncol 25:501-506, 2007

23. Spear MA, Jennings LC, Mankin HJ, Spiro IJ, Springfield DS, Gebhardt MC, et al: Individualizing management of aggressive fibromatoses. Int J Radiat Oncol Biol Phys 40:637-645, 1998

24. Wang B, Ma J, Jin H: Infantile intracranial aggressive fibromatosis: report of two cases with a review of the literature. Pediatr Neurosurg 48:181-186, 2012

25. West CB Jr, Shagets FW, Mansfield MJ: Nonsurgical treatment of aggressive fibromatosis in the head and neck. Otolaryngol Head Neck Surg 101:338-343, 1989

26. Yazici N, Yalçin B, Soylemezoğlu F, Cila A, Akalan N, Koksal Y, et al: Intracranial desmoid tumor with familial adenomatous polyposis coli. Pediatr Neurosurg 44:140-143, 2008

\section{Disclosures}

The authors report no conflict of interest concerning the materials or methods used in this study or the findings specified in this paper.

\section{Author Contributions}

Drafting the article: Li. Critically revising the article: all authors. Reviewed submitted version of manuscript: all authors. Approved the final version of the manuscript on behalf of all authors: Lew.

\section{Correspondence}

Sean M. Lew, Department of Neurosurgery, Children's Hospital of Wisconsin, 999 North 92nd St., Ste. 310, Milwaukee, WI 53226. email: slew@mcw.edu. 